\title{
Planning to plan: Real-time processes in the development of problem solving
}

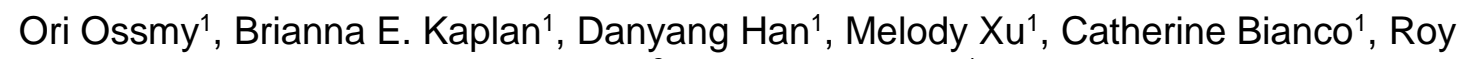 \\ Mukamel $^{2}$, Karen E. Adolph ${ }^{1}$ \\ ${ }^{1}$ Department of Psychology and Center of Neural Science, New York University, New York, NY, USA \\ ${ }^{2}$ School of Psychological Sciences and Sagol School of Neuroscience, Tel-Aviv University, Tel Aviv, Israel
}

\section{Acknowledgments}

This work was supported by NSF/SBE-BSF grant \#1627993 and DARPA grant N6600119-2-4035 to Karen Adolph and BSF grant \#2016858 to Roy Mukamel. 


\begin{abstract}
Problem solving is integral to goal-directed action-how to navigate an obstacle, open a latch, or grasp the handle of a tool. Traditionally, researchers focus on when in development children succeed at solving particular problems, but this outcome-oriented approach does not inform on underlying mechanisms. The current study focused on how children solve problems by examining the real-time interplay among planning components. We encouraged 3- to 5-yearolds $(N=32)$ and adults $(N=22)$ to grasp a hammer to pound a peg when the hammer handle pointed in different directions. We simultaneously recorded participants' movements, gaze, and neural activity using video micro-coding, motion-tracking, head-mounted eye tracking, and EEG. When the handle pointed toward their non-dominant hand, participants had to use an adaptive underhand initial grip to implement the tool efficiently. Younger children ("nonadaptive child solvers") used a habitual overhand grip that interfered with wielding the hammer, whereas adults and older children ("adaptive child solvers") used an underhand grip. Adaptive and nonadaptive solvers differed in when and where they directed visual attention when the handle direction was revealed, neural processing prior to movement (readiness potential), and the straightness of their hand path as they reached to grasp the hammer. Nonadaptive child solvers immediately used the habitual solution and only later gathered visual information, leaving insufficient time to form a plan before acting. We suggest that the development of adaptive and efficient problem solving depends on this real-time "tug of war" between habits and information gathering and processing.
\end{abstract}

\title{
$247 / 250$
}

Keywords: problem solving, action planning, child development, eye tracking, EEG 


\section{Introduction}

\section{Multi-Step Problem Solving}

Across tasks, ages, and cultures, problem solving is integral to functional motor behaviour-how to navigate an obstacle, open a latch, grasp the handle of a tool, and so on (Keen, 2011; Newell \& Simon, 1972). For some problems, task requirements are directly accessible in the scene (e.g., a large step is required to cross an obstacle in the path). Solving such directly perceptible motor problems begins in infancy. With age and experience, problem solving becomes more adaptive (appropriate action is selected to achieve the goal) and efficient (movements are faster, smoother, and more accurate) (Adolph \& Robinson, 2015; Lockman, Fears, \& Jung, 2018). Infants, for example, improve with age and experience in navigating obstacles such as bridges and drop-offs, reaching for objects varying in size and orientation, and catching balls moving at different speeds (Kretch \& Adolph, 2013a, 2013b; Lee, Liu, \& Newell, 2006; van Hof, van der Kamp, \& Savelsbergh, 2008; Witherington, 2005).

However, for many everyday problems, planning stretches far into the future and the required actions are not immediately available to perception: Must the latch be turned, pressed, or slid sideways prior to opening the door? Should the handle be grasped with an overhand or underhand grip prior to wielding the tool? In the case of such multi-step problems, people must plan their initial actions in anticipation of the end goal (Rosenbaum, Chapman, Weigelt, Weiss, \& van der Wel, 2012; Rosenbaum et al., 1990). Otherwise, reliance on habitual solutions may require ad hoc modifications to ongoing movements. For example, when flipping an upsidedown glass to fill it with water, adults initially grasp the glass with their thumb pointing down-a non-habitual, uncomfortable grip-so that after flipping it, their thumb comfortably points upward in a position more conducive to holding the glass for filling it with water. In short, solving such multi-step problems means that the latter part of the action (the part of the action not immediately available to perception, e.g. pouring water into the glass) influences the initial part (the part of the action that is directly available to perception, e.g. grasping the overturned glass); see Figure $1 \mathrm{~A}$ top panel.

In contrast to adults, when children flip a water glass, they begin with a habitual initial grip and end in an awkward thumb-down position (Knudsen, Henning, Wunsch, Weigelt, \& Aschersleben, 2012). More generally, in multi-step problems involving turning handles, flipping dowels, and implementing tools like spoons and hammers, infants and young children rely on habitual actions initially and thus fail to solve multi-step problems adaptively and efficiently (Comalli et al., 2016; Wunsch, Weiss, Schack, \& Weigelt, 2015); see Figure $1 \mathrm{~A}$ bottom panel. They resort to clumsy "trial-and-error" actions as they fumble around make online error corrections to accomplish the end goal. Across studies, children show developmental improvements in multi-step problem solving until about 10-12 years of age, when most attain adult-like levels; for reviews, see (Adolph \& Robinson, 2015; Keen, 2011; Willats, 1989; Wunsch, Henning, Aschersleben, \& Weigelt, 2013).

\section{Planning Adaptive Solutions}

Planning is essential to multi-step problem solving and thus plays a critical role in its development (Keen, 2011; Willats, 1989; Wunsch et al., 2013). Tool use is an important paradigm for the study of multi-step problem solving because successful implementation reflects planning for a distant goal. For example, when adults want to hammer a peg and the handle points toward their non-dominant hand, they plan two steps ahead by grasping the hammer with an underhand grip that allows for a smooth transition to a comfortable end-state grip (Comalli et al., 2016). Researchers agree that the behavioral solutions to motor problems (e.g., using an underhand vs. overhand grip) reflect multi-step action planning or lack thereof (Keen, 2011; Rosenbaum et al., 2012). The logic is simple: If the same tool is grasped differently depending on the actor's plan, then multi-step planning can be inferred from the way the tool was grasped. 
However, planning also involves processes that are not captured in easily observable behavioral solutions. Planning is a real-time cascade of perceptual, neural, and motor events (Ossmy, Han, Cheng, Kaplan, \& Adolph, 2020; Wong, 2015). It begins with gathering taskrelevant information; the perceptual information is then neurally integrated with the motor system; and finally, the action is produced. Despite the importance of planning in the development of problem solving, the question is still open as to how the interplay among perceptual, neural, and motoric components of planning relate to developmental improvements in problem solving.

A

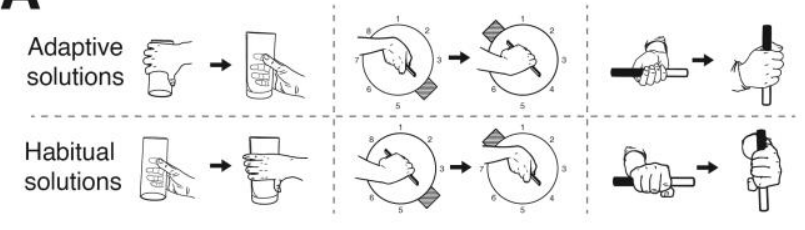

C

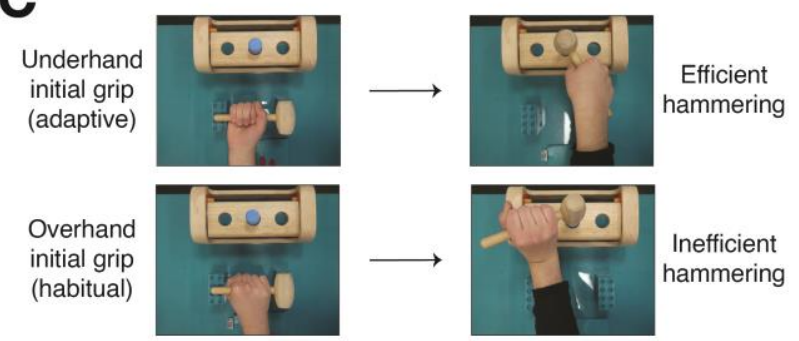

B

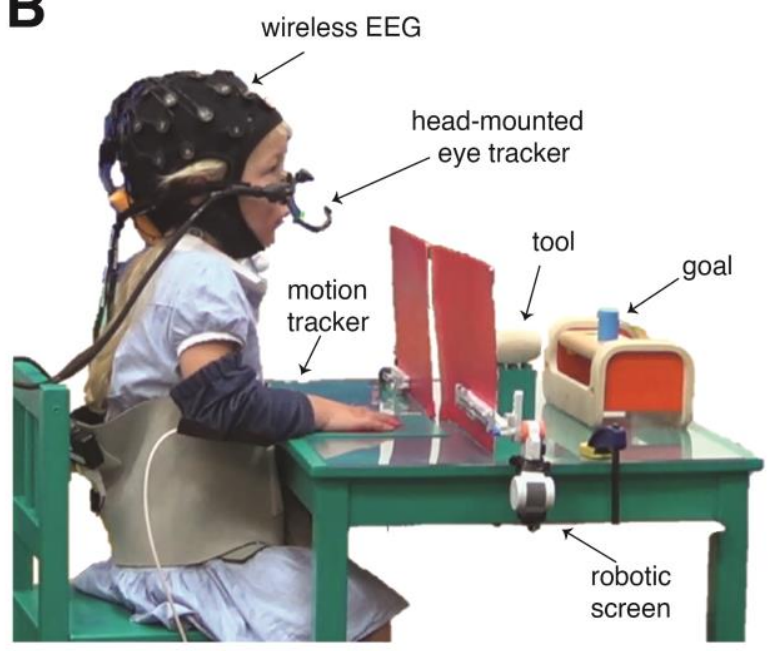

Figure 1. (A) Adaptive (top panel) vs. habitual (bottom panel) solutions to various motor problems. Drawings show hand configurations for initial grip (left of arrows) and subsequent implementation of the object (right of arrows). Examples of adaptive problem solving -an initial thumb-down grip allows for optimal thumb-up grip while filling the glass; a highly torqued initial grip allows for optimal end grip while turning the handle; and an initial underhand grip allows for optimal thumb-up grip while placing the dowel. The habitual solutions are not adaptive-initial thumb-up grip that leads to awkward thumb-down grip while filling the glass; minimally torqued initial grip leads to a highly torqued and awkward grip while turning the handle; and initial overhand grip leads to awkward thumb-down grip while placing the dowel. (B) Hammer-and-peg task. Participants sat in front of a robotic screen and were asked to wield a toy hammer to pound a peg. They wore a head-mounted eye-tracker, EEG cap, and motion tracking sleeve. (C) Initial grips and subsequent hammering on non-dominant-hand trials. Top: Underhand/radial grip leads to a comfortable position for pounding the peg. Bottom: Overhand/ulnar grip leads to an awkward position for pounding the peg.

\section{Current Study}

Here, we adopted a new integrative approach that combines interdisciplinary perspectives and methods from developmental psychology, neuroscience, and movement science. We recorded perceptual, neural, and motoric planning processes in real time as children and adults grasped a hammer to pound a peg. We tested children at an age (3 to 5 years) when they were sure to succeed in using the hammer-regardless of whether they did so adaptively or efficiently — but potentially lack adult-like multi-step planning. Participants wore a head-mounted eye tracker to record where they looked and when, an EEG cap to measure their neural activity during planning, and a motion tracker to record the details of their hand trajectory; video captured their initial grasp and success at pounding the peg (Figure 1B). 
We tested participants in "dominant-hand" trials, where the handle of the hammer pointed toward their dominant hand, and "non-dominant-hand" trials, where the handle pointed toward their non-dominant hand (Figure 1C). In the dominant-hand trials, adaptive problem solving would be evidenced by a habitual overhand grip, whereas in the non-dominant-hand trials, adaptive problem solving would be evidenced by an underhand grip (Figure 1C). We assessed efficiency based on how quickly and smoothly participants grasped the hammer with their dominant hand and whether their pounding was accurate (hit or missed the peg).

Based on previous work (Comalli et al., 2016), we expected problem solving to become more adaptive and efficient with children's age, and from children to adults. The important contribution of the current work, however, is the insights provided by our simultaneous recording methods into the typically hidden perceptual, neural, and motoric processes that underlie multistep planning. Our primary hypothesis was that age-related differences depend on developmental changes in the timing of each component in the real-time planning cascade. We expected that adaptive problem solvers would display smoother, more direct reaching trajectories in both conditions. We predicted that adaptive problem solvers would plan solutions ahead of time by starting the cascade early and maintaining the correct order of components (early fixations to task-relevant objects, neural differentiation of handle direction, and then movement onset), and nonadaptive problem solvers would rely on habitual overhand grips on non-dominant-hand trials and resort to online error correction because they start the cascade too late and display later components of the cascade before earlier ones.

\section{Results}

Participants contributed $M=58.20$ trials (21.14 dominant-hand trials, 37.05 nondominant-hand trials). Adults and children did not differ in total number of trials (Ms $=60.18$ and 56.84 respectively; $t(52)=1.04, p=.30$ ). Preliminary analyses showed no effects of sex, so sex was collapsed in subsequent analyses.

\section{Solutions to Multi-Step Problems Become More Adaptive and Efficient Over Development}

All participants were highly motivated by the hammering task and pounded the peg flat on every trial. They used initial overhand grips on dominant-hand trials, although children tended to be less consistent than adults, $M s=96.40 \%$ and $99.60 \%$ respectively, $t(52)=1.93, p$ $=.06$ (Figure 2, top panel). On non-dominant-hand trials, adults uniformly used initial underhand grips, $M=97.72 \%, S D=4.21$ (Figure 2, left-bottom panel), whereas children's grip choices were variable, $M=60.12 \%, S D=41.57 \%$ (Figure 2, right-bottom panel). Overall, adults performed more underhand grips than children on non-dominant-hand-trials, $t(52)=4.21, p<$ .00 . The proportion of underhand grips increased with children's age, $r(30)=.50, p<.00$ (Spearman correlation). These results confirm that participants became more adaptive in solving the hammering problem with development.

Children were also less efficient than adults on non-dominant-hand trials. In particular, children were slower to pound the peg flat compared to adults from the moment the trial started, $M s=6.61 \mathrm{~s}$ and 2.66s, respectively, $t(52)=8.09, p<.00$ (Figure S1A), and they were less accurate (i.e., showed more implementation errors where they missed the peg; see Video S1), $M s=7.71$ and 0.63 errors per session, respectively, $t(52)=7.63, p<.00$ (Figure S1B).

Moreover, children's efficiency depended on whether they were adaptive when they first grasped the hammer. Children who were more adaptive-performed more initial underhand grips on non-dominant-hand trials-were faster, $r(30)=-.49, p<.00$, and more accurate, $r(30)=$ $-.55, p<.00$. When children were not adaptive and used an initial overhand grip, speed and accuracy depended on whether they changed their grip after grasping the hammer. Children changed $79.88 \%$ of their overhand grips on non-dominant-hand trials. They were slower when they changed their grip compared to when they pounded the peg with an awkward grip, $M s=$ $7.69 \mathrm{~s}$ and $6.94 \mathrm{~s}$, respectively, $t(505)=2.06, p<.04$. But after changing grips, children tended to 
be more accurate than when they did not, $M s=0.27$ and 0.92 implementation errors, respectively, $t(505)=1.12, p<.07$.

Figure 2 and $\mathrm{S} 1$ show that children are significantly more variable than adults in the adaptiveness and efficiency of problem solving. To understand the sources of interindividual variability in children, we split them into two groups based on their initial grips. One groupadaptive child solvers -included all children who demonstrated adult-like problem solving $(N=$ 15). That is, they used the underhand grip on at least $82.50 \%$ of the non-dominant-hand trials (this criterion was determined by the minimum proportion of underhand grips in adults; Figure 2, bottom panel). The second group-nonadaptive child solvers-included the rest of the children $(N=17)$. Our subsequent analyses focused on differences in planning between adults, adaptive child solvers, and nonadaptive child solvers.
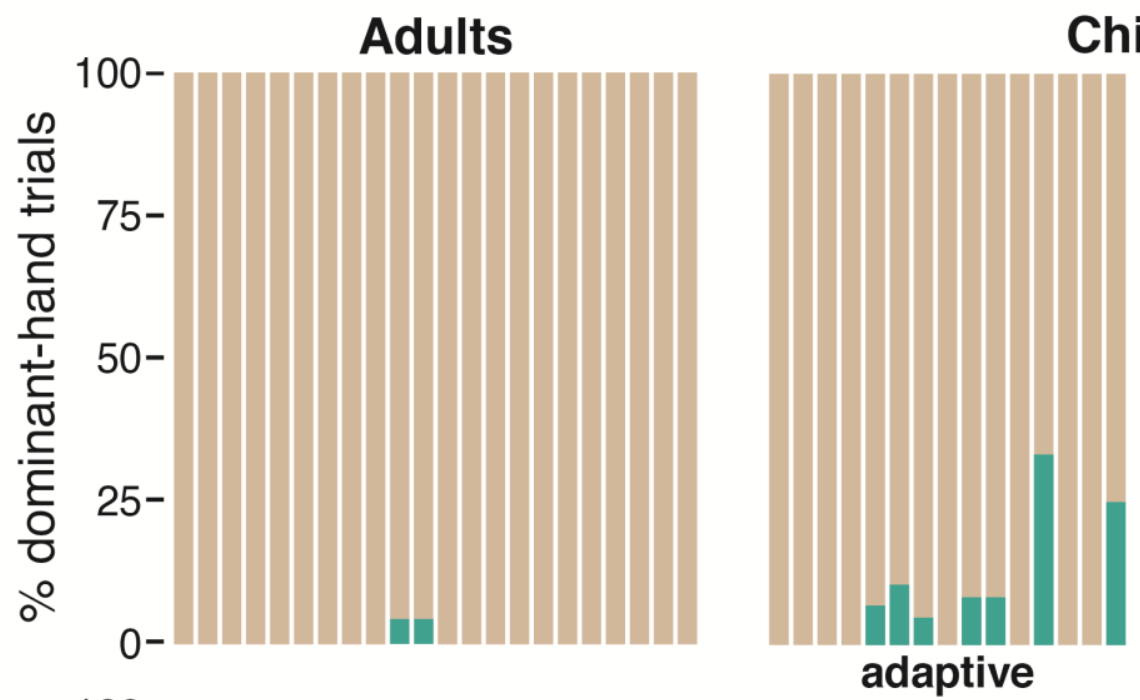

Children
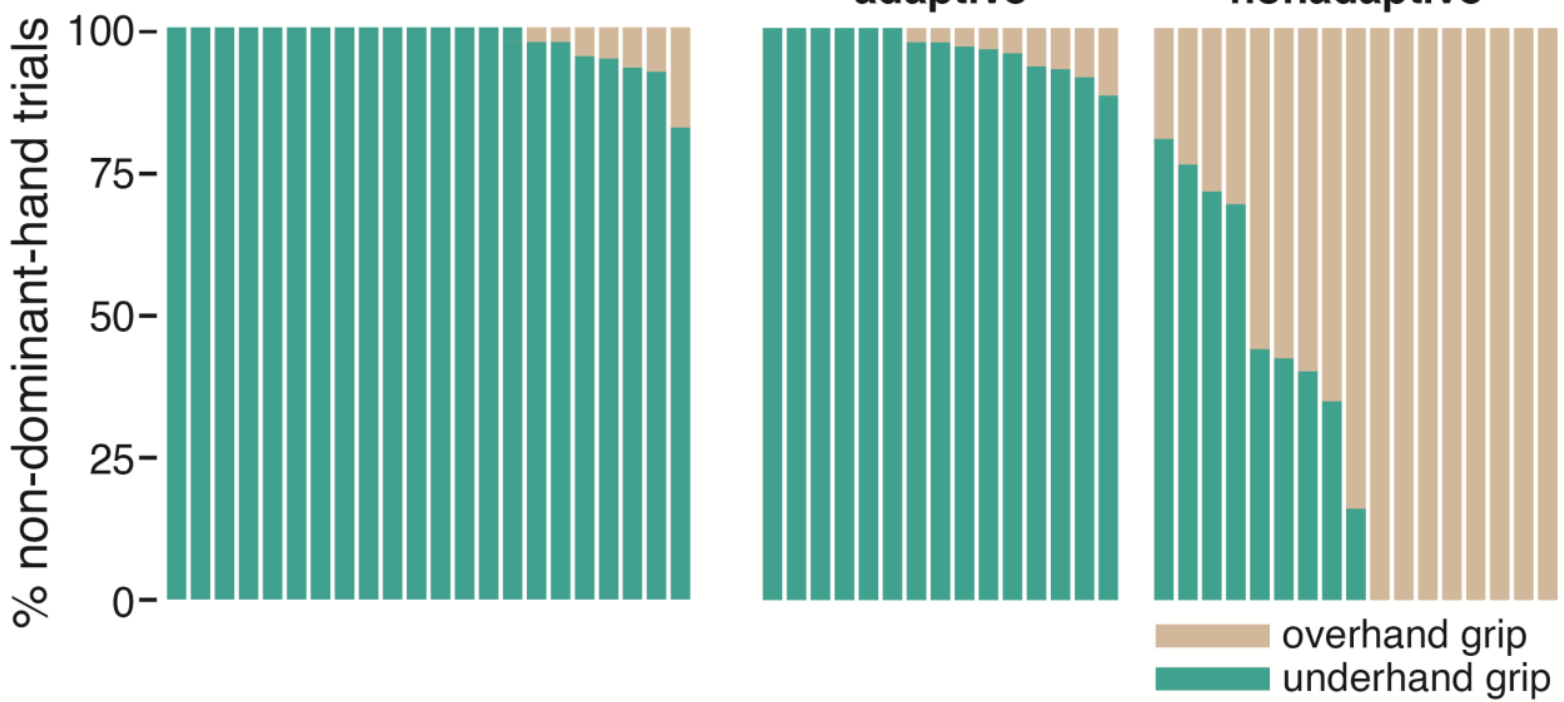

Figure 2. Primary measure of adaptive problem solving: Inter- and intraindividual variability in initial grip on dominant-hand trials and on-dominant-hand trials. In the non-dominant-hand trials, adults and 15 children (adaptive-child solvers) used underhand grips (>82.5\% of non-dominant-hand trials); 17 nonadaptive child solvers were more variable or simply continued to use the habitual, overhand grip regardless of handle direction. Each pair of bars (reading top to bottom) represents a single participant in the dominant- and non-dominant-hand trials. 


\section{Adaptive Problem Solvers Plan Ahead}

Adults, adaptive child solvers, and nonadaptive child solvers differed in when they planned their actions. Specifically, we evaluated differences in their reaching trajectories. Early differences in reach trajectories for dominant- versus non-dominant-hand trials indicate more planning ahead than differences at the last moment before grasping the hammer (or not at all). Figure $3 \mathrm{~A}$ shows the reach trajectories of one exemplar participant per group. The adult showed smoother movements and earlier differentiation of reach trajectories between dominant- and non-dominant-hand trials than did children. The adaptive child solver showed earlier differentiation than the nonadaptive child solver. A one-way ANOVA on the time to differentiate reach trajectories (see Methods) confirmed similar effects at the group level, $M \mathrm{~s}=77.46 \mathrm{~ms}$, $147.99 \mathrm{~ms}$, and $182.38 \mathrm{~ms}$ after movement onset for adults, adaptive child solvers, and nonadaptive child solvers, respectively, $F(2,53)=13.20, p<.00$ (Figure 3B). For the nonadaptive solvers, trajectory differentiation occurred after the moment of initial grip, so there was no trajectory differentiation while reaching. Sidak-corrected post hoc comparisons confirmed significant differences between each pair of groups, all $p s<.01$.
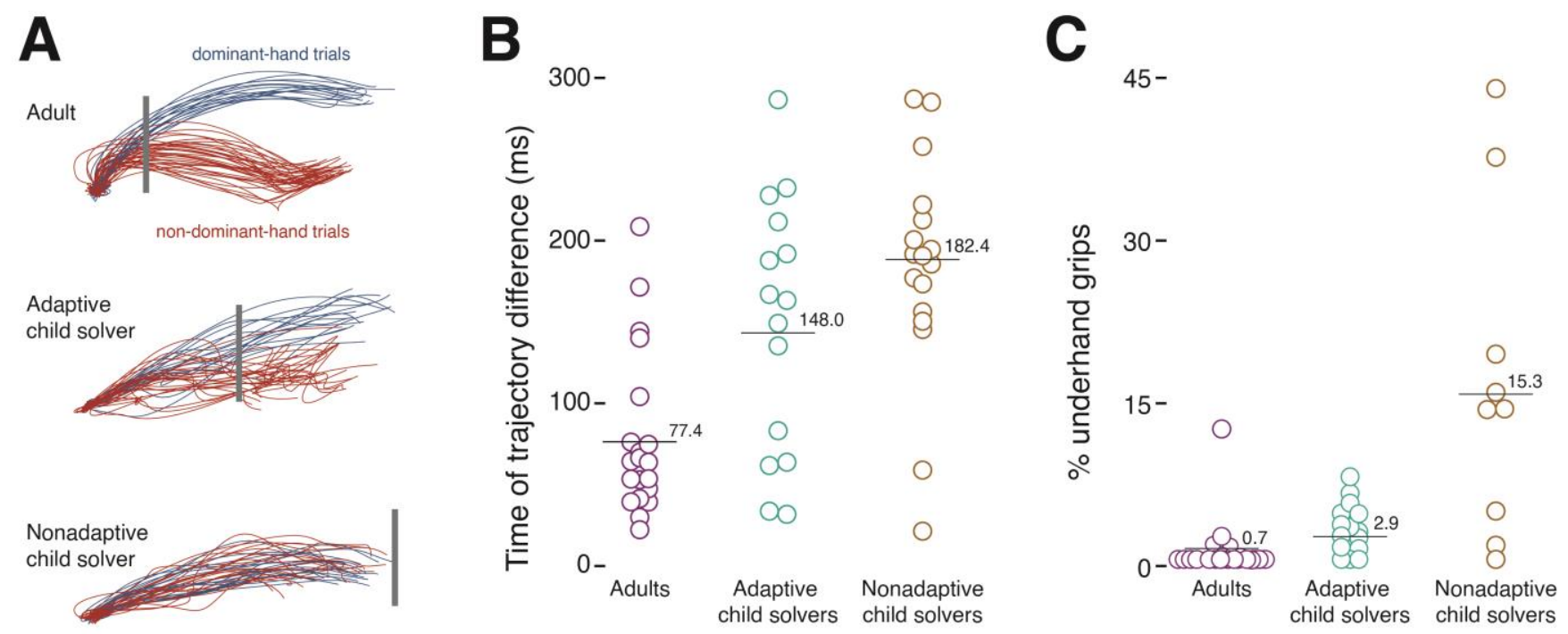

Figure 3. Measures of efficiency and early planning in adults, adaptive child solvers, and nonadaptive child solvers. (A) Motion-tracking shows reach trajectories for each trial in exemplar participants from each group. Adult exemplar shows more efficient (smoother, more consistent) reach trajectories than child exemplars. Moreover, the adult and adaptive child solver showed differentiation in reach trajectories in dominant-hand versus non-dominant-hand trials, whereas the nonadaptive child solver showed identical reach trajectories for both hammer orientations. Vertical lines indicate the moment of significant trajectory difference between conditions (line to right of nonadaptive child solver denotes no trajectory difference before grasping). (B) Time to differentiate reach trajectories between dominant- and nondominant-hand trials. Each symbol denotes the mean differentiation time for each participant. Horizontal lines denote group means. Adults showed earlier differentiation of reach trajectories, followed by adaptive child solvers, and finally nonadaptive child solvers. (C) Last-moment decisions (an index of inefficiency) to use underhand grips on non-dominant-hand trials. Nonadaptive child solvers showed more last-moment decisions (See Methods). Note, only 9 nonadaptive child solvers used an underhand grip.

We also found differences in how participants planned underhand grips on nondominant-hand trials. We identified each trial in which participants made last-moment grip decisions (i.e., began to reach and then changed direction to accommodate a switch from overhand to underhand); see Video S2. On the relatively few trials when nonadaptive child solvers used an underhand grip, they decided to do so at the last moment more often ( $M=$ 
$15.33 \%$ of underhand grips; see Methods for definition of last-moment decisions) than adaptive child solvers, $M=2.90 \%$, and adults showed even fewer last moment decisions, $M=0.69 \%$, $F(2,43)=11.56, p<.00$ (Figure $3 C$ ). Sidak-corrected post hoc comparisons confirmed significant differences between the nonadaptive child solvers and the other groups, $p s<.00$.

Planning Ahead Depends on the Real-Time Interplay between Planning Components

What factors drive the developmental improvements in planning? We examined the ratio between participants' movement time and planning time (see Methods). A low ratio means that participants "saved" movement time by investing time in planning before starting their movement. As shown in Figure 4, adults had the lowest movement-to-planning ratio, $M=3.65$, $S D=1.36$, and adaptive child solvers had a lower ratio than nonadaptive child solvers $(M \mathrm{~s}=$ 6.42 and 7.40, respectively). A one-way ANOVA on the movement-to-planning ratio confirmed a group effect, $F(2,53)=12.73, p<.00$; post hocs confirmed differences between all three groups, all $p s<.05$.

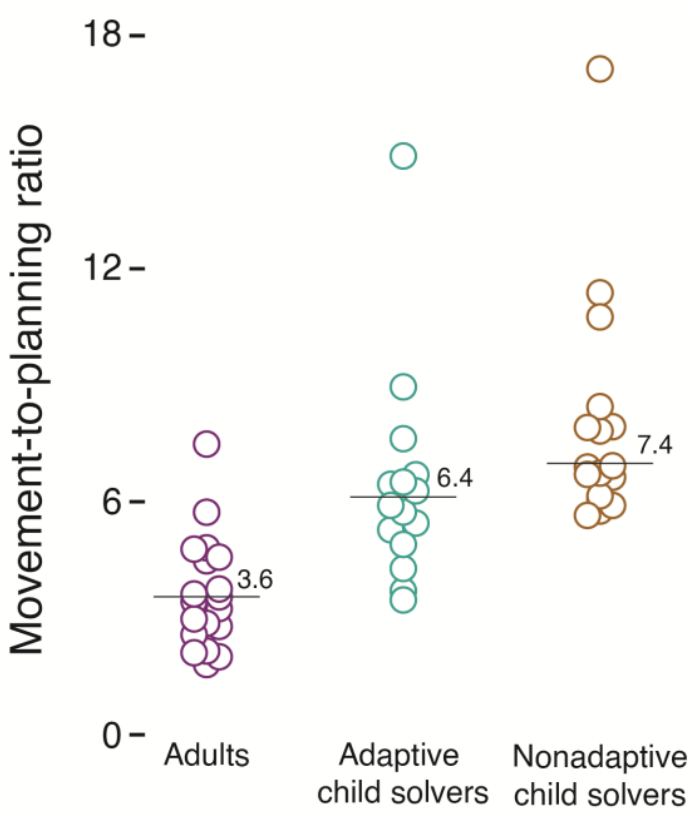

Figure 4. Movement-to-planning ratios in adults, adaptive child solvers, and nonadaptive child solvers. Each symbol denotes the mean ratio for each participant. Horizontal lines denote group means. Adults showed lower ratios (i.e., they spent relatively more time planning and less time moving), followed by adaptive child solvers, and finally nonadaptive child solvers.

More adaptive solvers gathered perceptual information about the action earlier than less adaptive solvers. From the moment the direction of the hammer was revealed, adults directed their gaze to the hammer faster, $M=336.73 \mathrm{~ms}, S D=138.47$, than adaptive child solvers, $M=$ $628.13 \mathrm{~ms}, S D=142.70$. Nonadaptive child solvers directed their gaze to the hammer much later, $M=839.73 \mathrm{~ms}, S D=196.68$. A one-way ANOVA confirmed a main effect for group, $F(2,53)=48.45, p<.00$; post hocs confirmed differences between all three groups, all $p s<.05$ (Figure 5A).

Moreover, adults mainly fixated the hammer before they began their movement $(M=$ $43.23 \%$ of the time interval between screen opening to movement onset, $S D=20.05$; Figure $5 B)$ rather than the peg $(M=13.43 \%, S D=14.91)$ or their hand $(M=0.88 \%, S D=1.51)$. We found similar behaviors in adaptive child solvers, but to a lesser extent $(M=25.88 \%, S D=$ 13.75 on hammer; $M=8.05 \%, S D=3.66$ on peg; $M=3.68 \%, S D=2.99$ on hand), whereas 
nonadaptive child solvers did not fixate on any particular area of interest $(M=14.03 \%, S D=$ 6.55 on hammer; $M=12.45 \%, S D=8.29$ on peg; $M=10.11 \%, S D=6.06$ on hand). $A 3$ (groups) $\times 3$ (regions of interest) mixed model ANOVA confirmed main effects for group $F(2,51)=12.47, p<.00$, and region $F(1,51)=27.70, p<.00$, and an interaction between group and region $F(2,51)=7.47, p<.00$. Sidak-corrected post hoc tests confirmed higher percentages in looking at the hammer in adults than adaptive child solvers, and higher percentages in adaptive child solvers than nonadaptive child solvers, all $p s<.05$, and no group differences in looking at the hand or the peg, all ps > .05 (Figure 5B).

These findings were further supported by participants' distribution of attention (heat map in Figure $5 \mathrm{C}$ ). Adults looked at $M=9.27 \%$ of pixels in the video frames, adaptive child solvers looked at $M=15.81 \%$ of the pixels and nonadaptive child solvers looked at $M=20.85 \%$ (see Video S3). A one-way ANOVA confirmed a main effect for group, $F(2,51)=37.05, p<.00$. Sidak-corrected post hoc tests showed a broader distribution of attention (see Methods) in nonadaptive child solvers compared to the other two groups, all $p s<.05$ (Figure 5D).
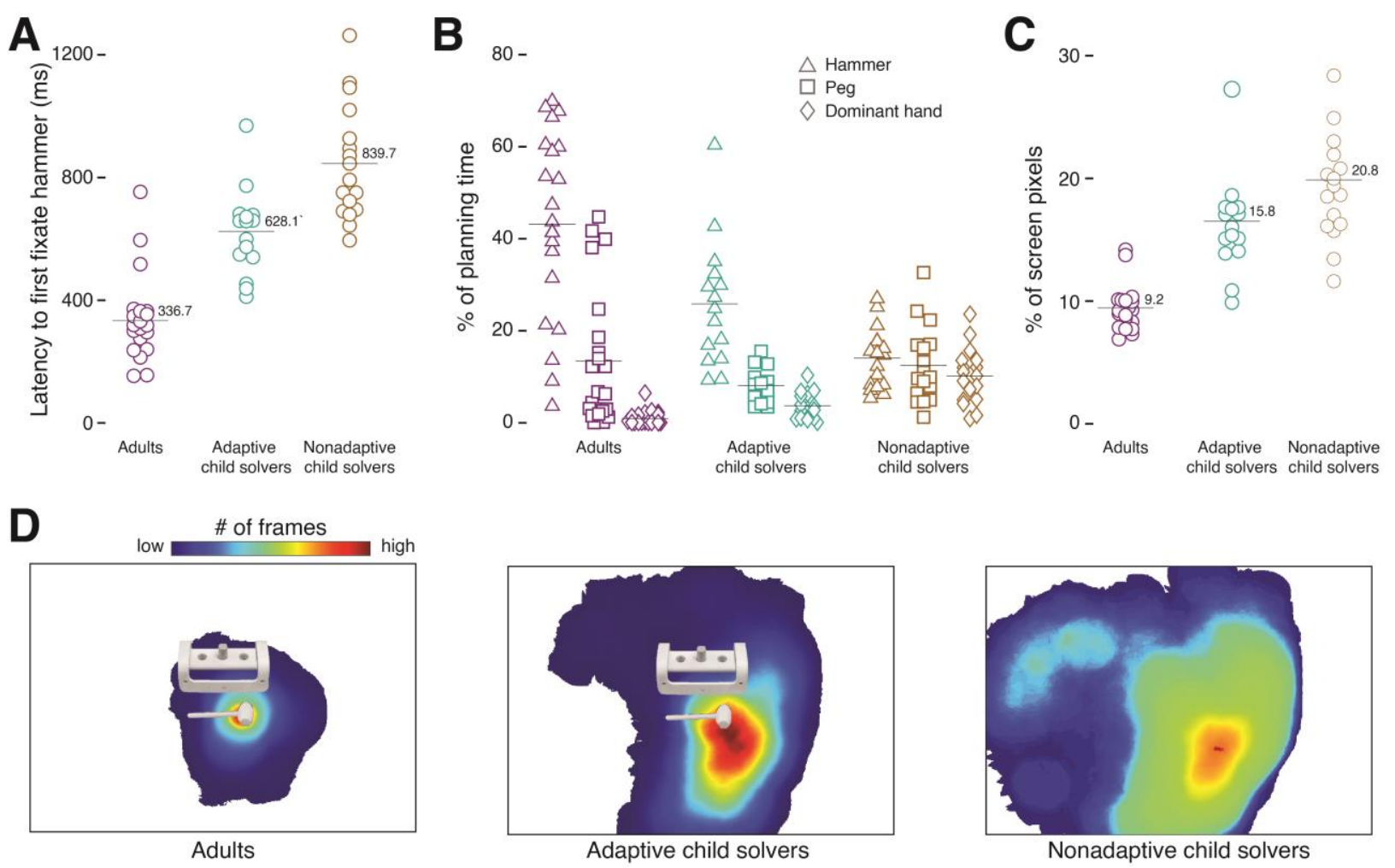

Figure 5. Visual information gathering prior to moving the hand in adults, adaptive child solvers, and nonadaptive child solvers. (A) Latency to fixate the hammer after the screen lifted. Each symbol denotes the mean latency for each participant. Horizontal lines denote group means. (B) Looking to ROls (hammer, peg board, dominant hand) during the period from screen opening to moving the hand (i.e., percent of planning time). Each symbol denotes the mean percent of planning time for each participant. Horizontal lines denote group means. (C) Percent of pixels fixated during the period from screen opening to moving the hand. Smaller percentages indicate more focused attention. Each symbol denotes the mean percent of pixels for each participant. Horizontal lines denote group means. (D) Heat maps showing dispersion of visual attention accumulated across each group. Colored regions denote areas fixated. Hotter colors denote longer accumulated fixation times. Heat maps in (D) cover more area than individual data in $(\mathrm{C})$ because often participants' fixation locations did not overlap. 
Neural processing before movement onset also differed between the groups. Because non-dominant-hand trials required a different action plan then dominant-hand trials, we analyzed differences between conditions in readiness potential-event-related potential (ERP) over the sensorimotor cortex prior to movement known to correspond to motor planning (see Methods). Figure 6 shows that the readiness potential significantly differed in adults at $M=473 \mathrm{~ms}, S D=$ 70.12 , before movement onset. Adaptive child solvers shows significant neural differentiation at $M=242 \mathrm{~ms}, S D=132.74$, before movement. For the nonadaptive child solvers, readiness potential did not differ between conditions.
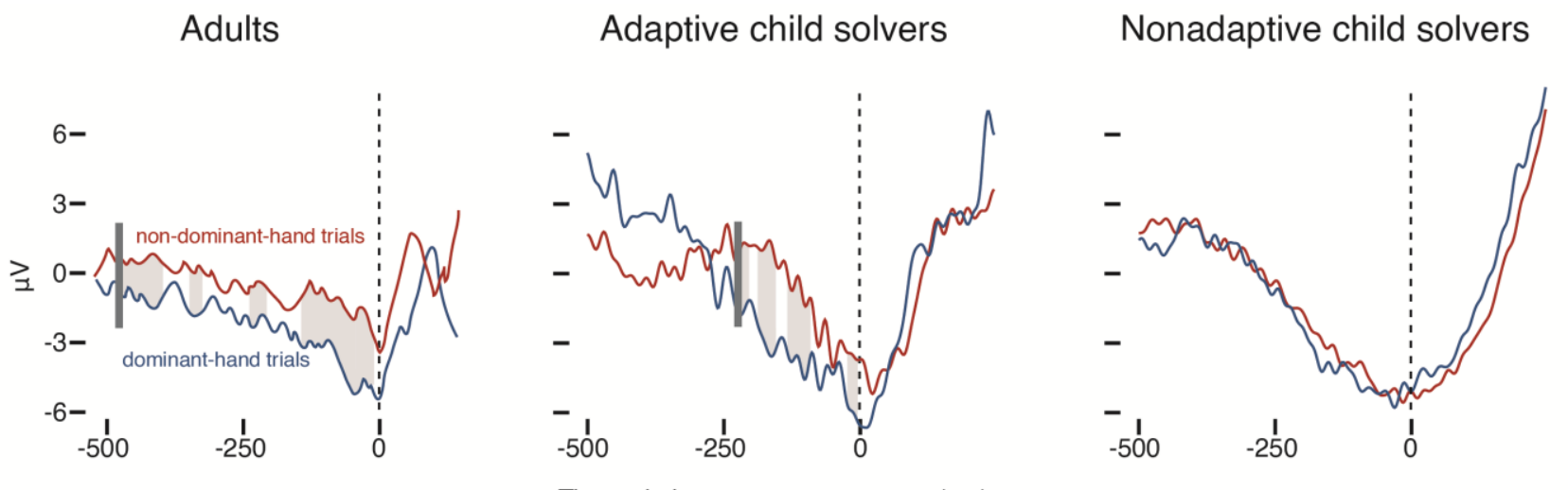

Time relative to movement onset $(\mathrm{ms})$

Figure 6. Neural differentiation (EEG) prior to moving the hand. Average readiness potential in dominantand non-dominant-hand conditions in adults, adaptive child solvers, and nonadaptive child solvers. Lightgray regions denote significant differences between conditions. Vertical dark-gray lines denote the onset of neural differentiation. Vertical dashed lines denote the moment of movement onset.

\section{Discussion}

Expanding on prior work that used multi-step tool use as a model system to investigate the development of problem solving (Comalli et al., 2016; Wunsch et al., 2013), we found that adults were more adaptive than children, older children were more adaptive than younger children, and nonadaptive child solvers showed high intra- and interindividual variability. However, prior work could only speculate about the causes of children's nonadaptive, inefficient performance. Here, we used a novel combination of simultaneous recording methods-headmounted eye tracking, EEG, motion tracking, and third-person video-to transcend mere speculation.

The current study is the first to identify the real-time factors that underlie developmental improvements in adaptiveness and efficiency. As we predicted, more adaptive problem solving led to more efficient performance. And as we hypothesized, adults and adaptive child solvers were adaptive and efficient because they started the real-time planning cascade early and maintained the correct order of planning components. As summarized in Figure 7, adaptive problem solvers gathered and processed perceptual information before they began moving; that is, they displayed early visual fixation to the hammer and early neural differentiation of the motor plan. As a consequence, adaptive solvers showed early movement differentiation between overhand and underhand grips. In contrast, nonadaptive child solvers were less adaptive and efficient because they started the cascade too late and thus displayed later components of the cascade before earlier ones or failed to display all the components (i.e., failed to differentiate perceptual information via neural activity or reach trajectories). Planning in nonadaptive solvers went awry from the first reveal of the tool, and errors consequently propagated through the system. 


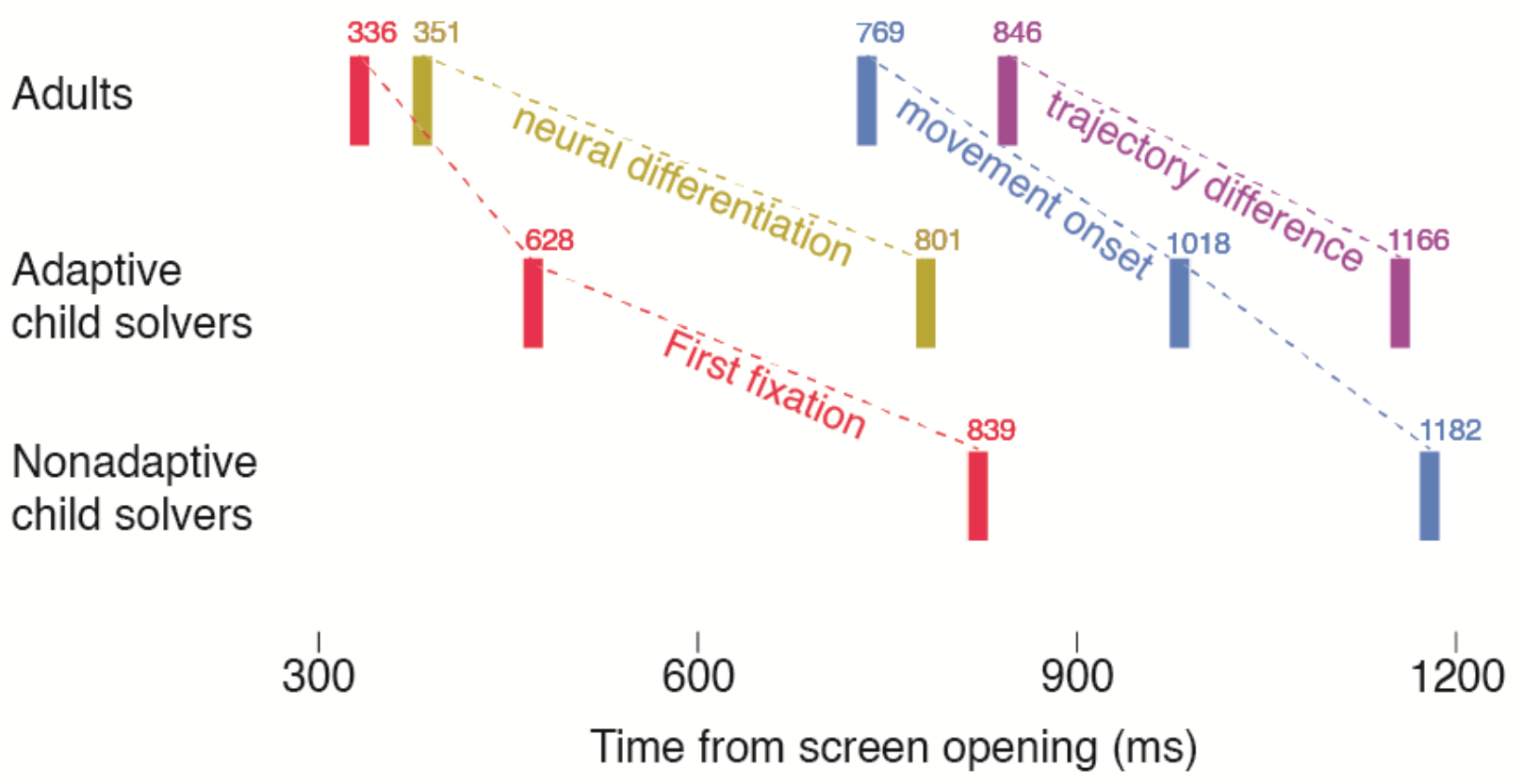

Figure 7. Timing of the real-time planning cascade starting when the screen opened and ending at grasp of the hammer handle. Vertical solid lines denote group averages for each component. Dashed lines facilitate comparisons between groups. Adults displayed earlier visual fixation to the hammer, earlier neural differentiation of the handle orientation, earlier movement onset, and earlier differentiation of reach trajectories than adaptive child solvers. The adaptive child solvers, in turn, displayed more components of the cascade and earlier registration of displayed components than nonadaptive child solvers.

\section{A Tug of War Between Habitual Solutions and Early Planning}

We suggest that adaptive, efficient problem solving emerges from developmental improvements in a real-time "tug of war" between habits and information gathering/processing. All things being equal, children and adults rely on habitual solutions to motor problems. These habits compete with new information. Thus, early gathering and processing of perceptual information about task constraints provided adults and adaptive child solvers with sufficient time to determine whether the habitual overhand grip was appropriate for the specific situation (i.e., direction of hammer). On non-dominant hand trials, it gave them time to determine that the habitual grip was not optimal and a non-habitual-initially awkward — underhand grip offered a more adaptive and efficient solution. Thus, adaptive solvers generated a plan on the fly. They were able to do this because they looked at the task-relevant areas early enough to discover the task demands and give their brains sufficient time to process the perceptual information and to form a plan before acting. In contrast, nonadaptive child solvers were slow to gather taskrelevant information, leaving insufficient time to process the information in their brains before movement onset. This led nonadaptive solvers to rely on their habitual solution and resort to online error correction.

This real-time tug of war during action planning is in line with computational cognitive research that distinguishes between model-free and model-based decision-making strategies (Daw, Niv, \& Dayan, 2005; Nussenbaum \& Hartley, 2019). With a model-free strategy, agents repeat actions that were frequently rewarded in the past. With a model-based strategy, agents acquire an internal representation of the task to evaluate possible actions. Previous research found model-free decision-making strategies in both children and adults, whereas a modelbased strategy emerges only in adolescence and increases with age (Decker, Otto, Daw, \& Hartley, 2016). Similarly, we found that nonadaptive child solvers repeated a habitual overhand 
grip that presumably worked for them in most everyday grasping tasks (akin to a model-free strategy), whereas adults and adaptive child solvers learned to initiate an early planning cascade so they could evaluate possible actions rather than simply execute the habitual one (akin to a model-based strategy). Our findings suggest that a developmental shift from a modelfree to model-based strategy is not only a property of higher-level cognition such as decision making, cognitive flexibility, executive function, and self-regulation. It is also critical for perceptual-motor planning, and the shift depends on the age when children learn the optimal time to gather and process perceptual information. Further research on the connections between perceptual-motor planning and computational cognitive research is required to address the gap between motor problem-solving skills and higher cognitive functions.

\section{Planning to Plan: The Role of Perception in Early Action Planning}

Ours is not the first study, of course, to investigate action planning. Most empirical and computational work argues that actors acquire the ability to plan ahead when they learn to maintain a cognitive representation of future action consequences based on the initial task parameters (Land, Volchenkov, Bläsing, \& Schack, 2013; Stockel, Hughes, \& Schack, 2012). In other words, actors learn to plan their actions prior to commencing a movement when they learn to anticipate the outcome of a specific action given the environmental characteristics and requirements in which the action is being performed. However, cognitive researchers typically pay little attention to the way perceptual information is gathered. In prior work on the development of multi-step problem solving, information about task parameters is taken for granted, as if the environment is already known before movement commences (Fuelscher, Williams, Wilmut, Enticott, \& Hyde, 2016; Toussaint, Tahej, Thibaut, Possamai, \& Badets, 2013).

By investigating the internal perceptual, neural, and motor processes that underlie planning, we show this is not the case. Participants did not simply "know" all the relevant information about the hammering task before the screen lifted at the start of each trial. How could they? They needed to deliberately point their eyes to the visual information that would allow them to plan their actions. Children's deficit in planning ahead results from deficits in "planning to plan." Looking is itself an action that requires planning (Gibson, 1979), and nonadaptive child solvers did not plan when and where to look. This finding expands on recent work showing that adults plan their actions better than children during object fitting because adults gather information about the object and aperture earlier than do children (Ossmy et al., 2020). Moreover, informing children about their action consequences does not necessarily improve their planning. For example, when posed with solving multi-step motor problems such as flipping a dowel or an overturned water glass (Figure 1A), feedback about the consequence of the movement (flashing lights) did not improve children's motor planning (Knudsen et al., 2012).

\section{The Value of a Real-time Approach to the Development of Planning}

Typical research practices limit theoretical conclusions about the development of planning. In most studies, each child receives only one trial or perhaps a few trials; thus planning must be assessed based on the percent of children at each age who show efficient versus inefficient planning (Wunsch et al., 2013). However, small numbers of trials and group averages provide little information about the causes of intra- and inter-individual variability in children's planning.

Moreover, researchers typically evaluate planning efficiency based on the outcome of selected actions (Rosenbaum \& Feghhi, 2019) or use reaction time (the time for the actor to start moving) as a proxy for unspecified processes that occur before movement begins (e.g., Krajenbrink, Lust, Wilson, \& Steenbergen, 2020). However, focus only on movement outcomes limits potential explanatory mechanisms. For example, Rosenbaum and Feghhi (Rosenbaum \& Feghhi, 2019) argue that children gradually adopt more adult-like planning because their joints 
become stiffer as they mature, which reduces the number of movement strategies and makes the most efficient strategy more obvious. Biomechanical changes may indeed be important in the development of planning, but other factors are also likely contributors. Although reaction time reflects processes prior to movement, shorter reaction times do not indicate what process improved in planning (Wong, 2015).

Our multi-method approach demonstrates the promise of measuring the real-time interplay among perceptual, neural, and motor processes during planning over many trials for each child, before and after movement onset. We obtained 60 trials from each child, used a novel combination of real-time recording technologies, and examined planning processes prior to movement (gaze locations, neural differentiation of readiness potential) and after movement onset (differentiation of reach trajectories and grip choices). Our findings pave the way for research on the causes of nonadaptive, inefficient problem solving in individual children. Some children may fail to gather the relevant perceptual information before acting. As Ossmy and colleagues (Ossmy et al., 2020) put it, they leap before they look. Other children may fail to process the information rapidly and adequately. In other words, the problem is not a lack of perceptual information but rather what children do with the information they obtain (Shutts, Ornkloo, von Hofsten, Keen, \& Spelke, 2009). And even when children obtain the relevant information before implementing the solution and process it quickly and appropriately, they trail behind adults because they lack adult-like motor dexterity (Rosenbaum \& Feghhi, 2019).

One component of the planning cascade can affect downstream components, and some children may suffer from deficits on all counts. Testing these failures at the individual level may have translational implications for understanding disorders related to deficits in action planning and thereby support interventions to foster problem solving in individual children. Presumably, by knowing the source of deficits in individuals, interventions can be tailored to their specific needs.

\section{Conclusions}

The current study focused on how children solve problems by examining the real-time interplay among planning components. Our uniquely powerful combination of head-mounted eye tracking, EEG, motion tacking, and video indicates that each component of a planning cascade contributes to planning efficiency, and developmental changes in adaptive problem solving are driven by changes in the onset of the planning cascade and the timing of its components. Our findings provide new insights into the role of perception in action planning and suggest that adaptive and efficient problem solving depends on a temporal competition between habitual motor actions and the gathering/processing of perceptual information.

\section{Methods}

\section{Participants}

We tested 32 children from 3.13 to 4.96 years of age $(M=4.03$ years; 14 girls $)$ and 22 adults ( $M=21.90$ years; 13 women). Data from 9 additional children and 4 adults were excluded because the technology malfunctioned $(n=9)$, children refused to wear all the technology $(n=3)$, or the participant was ambidextrous $(n=1)$. Children were recruited from maternity wards at local hospitals when they were born. Adults were recruited through word of mouth. All participants had normal vision (or wore contact lenses); none had cognitive deficits or neurological problems. Participants received a robot toy, photo magnet, and tote bag for participation. With participants' permission, their videos and demographic data are shared with authorized researchers in the Databrary library (https://nyu.databrary.org/volume/434).

We determined children's hand dominance based on parents' report of the hand children used to brush their teeth, cut with scissors, and hold a spoon or fork, and laboratory observations of the hand children used to cut with scissors and draw a line (two trials with each task). Twenty-nine children were right-handed, and 3 were left-handed based on uniform 
responses across every parent report and observational measure. Eighteen adults were righthanded and 4 were left-handed.

Due to technical issues (children moved before the robotic screen opened, EEG data were noisy, or recording devices malfunctioned), $M=3.72$ trials per participant were excluded from the EEG and motion tracking analyses.

\section{Procedure}

Participants' task was to pound a peg with a wooden hammer (12.5-cm long handle, cylindrical hammerhead with a length of $6.5 \mathrm{~cm}$ and a face diameter of $2.5 \mathrm{~cm}$ ). The peg (2.5-cm diameter) protruded $5 \mathrm{~cm}$ above the pegboard and was painted blue to contrast with the wooden pegboard (Figure 1B). Each 60 -minute session began by showing the head-mounted eye tracker, EEG cap, and motion tracking sleeve to caregivers and children. To motivate children to wear the recording devices, we told them it was a "robot costume," and presented children with the robot toy they would take home. Then, we fit participants with the recording devices and collected calibration data for the eye tracker. We put an oven mitt on participants' non-dominant hand to prevent them from using it to grasp the hammer (https://nyu.databrary.org/volume/707).

For the hammering task, participants sat at a small table facing the experimenter and a robotic screen (Figure 1B and https://nyu.databrary.org/volume/707). The screen (LEGO Mindstorm NXT) could open and shut to control when the hammer and pegboard were revealed and to facilitate synchronization among recording devices. The experimenter showed participants the hammer and peg and told them to "pound the peg with the hammer." The hammer rested on two blocks $16.5 \mathrm{~cm}$ apart so that participants could easily use either an overhand or underhand grip to grasp the handle.

Before each trial, the screen was closed and participants placed their dominant hand on a red handprint on the table. Trials began when the screen opened to reveal the handle of the hammer pointing toward participants' dominant or non-dominant hand. Children completed 60 hammering trials presented in 4 blocks of 15 trials -5 dominant-hand trials, where a habitual overhand grip was most efficient, and 10 non-dominant-hand trials, where an underhand grip was most efficient (Figure 1C), with presentation order pseudo-randomized. Between trial blocks, participants completed engaging filler tasks and children received stickers to maintain motivation.

\section{Recording Devices}

Third-person cameras. Sessions were videotaped at $30 \mathrm{fps}$ from overhead and side views so that all actions were visible. An additional magnified side view of the hammer clearly captured the initial grip. Camera views were synchronized and mixed into a single video online, which was then synced offline with the eye-tracker videos for ease of video coding and data processing.

Motion tracker. A magnetic motion-tracking sensor (Ascension, 3D Guidance trakSTAR) with a long-distance transmitter $(144 \mathrm{~cm})$ was attached to participants' dominant hand to record their precise movement trajectories $(500 \mathrm{~Hz})$ and to synchronize the EEG data with the video data. The sensor was placed inside a "sleeve" that held all the trailing wires and positioned the sensor on participants' dominant wrist).

Head-mounted eye-tracker. As in previous work (Franchak, Kretch, Soska, \& Adolph, 2011; Ossmy et al., 2020), participants wore a Positive Science head-mounted eye tracker (www.positivescience.com) so that we could record where they looked (Figure 1B). The equipment consists of child- or adult-sized eyeglass frames, a transmitter, and a battery pack (total weight $375 \mathrm{~g}$ ). We attached the headgear to the adjustable EEG cap for stability and connected it via wire leads to a computer on the far side of the room. Participants also wore a wide rubber belt with Velcro patches to secure the leads, transmitter, and battery pack to their back (Figure 1B and https://nyu.databrary.org/volume/707). The eye tracker includes two small 
cameras mounted on the eyeglass frames-one to record the right eye (illuminated with an infrared emitting diode), and one to record the scene $\left(54.4^{\circ} \times 42.2^{\circ}\right.$ field of view). We used PSEyeCamera to monitor both videos online. To calibrate the tracker, seated participants fixated on a display board placed $45^{\circ}$ from their eyes to capture the entire table as the scene. Yarbus software was used to sync the videos from the two cameras offline. We then calibrated the data offline by manually indicating the target locations. The software superimposes a $4^{\circ}$ radius circular cursor on the composite video to denote the point of gaze on each video frame with a spatial accuracy of $\sim 2^{\circ}$.

EEG. Participants wore a child- or adult-sized, wireless, 32-channel EEG cap (Neuroelectrics, ENOBIO 32, $500 \mathrm{~Hz}$ ). Data were recorded from 32 scalp electrodes at locations of the extended 10-20 system and from two electrodes on the right mastoid. The single-ended voltage was recorded between each electrode site and CMS/DRL electrodes. In five children, we recorded only 4 electrodes due to technical malfunction.

\section{Data Coding}

Coders used Datavyu (www.datavyu.org), a computerized video coding tool, to record the duration of particular events and the presence or absence of specific behaviors. To ensure inter-observer reliability, a primary coder scored $100 \%$ of each participant's data, and a second coder scored $25 \%$. Coders agreed on $99 \%$ of initial and end grips ( $\kappa s=.96$ and .98 , respectively, $p s<.00), 97.53 \%$ of implementation errors ( $\kappa=.93, p<.00), 97.66 \%$ of lastmoment grip decisions $(\kappa=.76, p<.00)$. Correlation coefficients between the two coders for coding reaction, movement, and looking times were $r s>.93, p s<.00$. Disagreements were resolved through discussion.

Initial and end grips. Coders scored participants' initial grip (first grasp of the hammer; Figure 1B) and end grip (how they held the hammer when they pounded down the peg) as overhand (palm down) or underhand (palm up) and as radial (thumb facing hammerhead) or ulnar (thumb facing away from hammerhead). For the end grip, coders also scored the handle orientation (whether participants held the hammer handle horizontally or vertically relative to the table). Changes from the initial to end grip reflect changes after grasping the hammer.

Planning time and movement time. To score participants' "planning time," coders identified the first video frame when the screen lifted and the first video frame when participants lifted their hand from the table (i.e., their reaction time). Participants' movement time was the duration between the first video frame when they lifted their hand until the first frame when the hammer touched the peg.

Last-moment grip decision. Coders scored whether participants changed their decision about their initial grip part way through their initial reach. Coders considered a trial to have a last-moment grip decision if there was at least one frame in which they moved their hand backward before touching the hammer during their initial reach (Video S2).

Implementation errors. Coders scored how many times the participant missed the peg on each trial (hammer did not touch the peg).

Gaze location. Based on the location of the gaze cursor in the eye-tracking video, coders scored every fixation to 3 regions of interest (ROIs): the hammer, peg board, or dominant hand. Gaze location was coded from screen opening to movement onset.

\section{Motion tracking: Data Processing and Analysis}

Kinematic data were analyzed using the MATLAB Signal Processing toolbox. We compared the reaching trajectories for dominant-hand and non-dominant-hand trials from movement onset until we identified the first moment when the trajectories differed. Preprocessing included a notch filter at $50 \mathrm{~Hz}$ and a band-pass 4th order Butterworth filter (5$500 \mathrm{~Hz}$ ).

We determined differentiation in reaching trajectories by calculating the 3D Euclidean distance between locations of the hand at each time point from movement onset between each 
pair of trials. If the distance between the locations of the hand at a specific time point in the same condition was significantly smaller than the distance between the locations of the hand at the same time point across different conditions, we considered that time as the moment of trajectory differentiation. Because there were unequal numbers of dominant- and non-dominanthand trials, we randomly selected trials from each condition according to the minimum number of trials across conditions and calculated unequal variance $t$-tests on the selected trials. We repeated the selection process 1000 times. We corrected for multiple comparisons by controlling the False Discovery Rate (FDR; Benjamini \& Hochberg, 1995) with a threshold of $\mathrm{q}(\mathrm{FDR})<.05$.

\section{Eye tracking: Heat Maps and Distribution of Attention}

We used a novel digitizing method for head-mounted eye tracking to record moment-tomoment changes in participants' gaze from screen opening to movement onset. Using a custom-built MATLAB software, coders manually digitized the location of the hammer and the peg at each frame of the eye-tracking video. The gaze location was obtained from the eye tracker and was verified by human coders. We used the $x y$ coordinates of the hammer and peg to rotate, shift, and scale the location of the gaze, hammer, and peg in each frame such that all frames were aligned with the hammer located at the center and the peg located above the hammer. This procedure allowed us to compare looking patterns around the task-relevant objects in the scene across frames and participants, although their location changed constantly with the head-mounted eye tracker (unlike desk-mounted eye trackers, where locations are fixed).

Based on the aligned data, we generated looking heat maps for each participant and calculated the distribution of visual attention around the scene from screen opening to movement onset. Heat maps were created by dividing the number of video frames each pixel was fixated (within a 75-pixel radius of participants' gaze location to account for detection resolution) across all trials and participants in the group by the total number of video frames. The distribution of attention was calculated at the trial level by counting the number of pixels that were fixated (or were within the 75-pixel radius) during the pre-movement interval of each trial.

\section{EEG: Data Processing and Analysis}

EEG data were analyzed offline using the EEGLAB tool for MATLAB (Delorme \& Makeig, 2004). Raw data were band-pass filtered offline between 1 and $40 \mathrm{~Hz}$ (Butterworth filter, $24 \mathrm{db}$ ), and re-referenced offline to the digital average of the two mastoids. Deflections from eye movements and blinks were corrected using independent component analysis. Epochs were extracted trial by trial for each time interval, with $0-1500$ ms prior to the screen lifting as the baseline.

For removing ocular artifacts, we used independent component analysis (ICA) implemented in the EEGLAB toolbox. Ocular ICA components (range across subjects: 1-2 components) were identified by visual inspection and deleted from the global signal. Noisy trial epochs (exceeding $\pm 250 \mu \mathrm{V}$ range) at FCz, Cz, CPz, FC1, FC3, FC2, FC4, C1, C3, C2, C4, $\mathrm{CP} 1, \mathrm{CP} 2, \mathrm{CP} 4$ channels were identified in the raw data and rejected from the analysis.

We tested differences between dominant- and non-dominant-hand trials in readiness potential-the average event-related potential before movement recorded from frontal and central regions that corresponds to action planning (Aridan, Ossmy, Buaron, Reznik, \& Mukamel, 2018). For each participant, we calculated the signal in the time window $500 \mathrm{~ms}$ prior to movement onset at components with activity over the contralateral primary motor cortex to the executing effector (components were identified by visual inspection). We then compared the signal in the dominant- and non-dominant-hand trials from movement onset backward until we identified the first moment when the signals significantly differed. To address the unequal number of dominant-hand and non-dominant-hand trials, we performed similar significance procedures as in the motion-tracking analysis. 


\section{References}

Adolph, K. E., \& Robinson, S. R. (2015). Motor development. In L. Liben \& U. Muller (Eds.), Handbook of child psychology and developmental science (7th ed., Vol. 2 Cognitive Processes, pp. 113-157). New York: Wiley.

Aridan, N., Ossmy, O., Buaron, B., Reznik, D., \& Mukamel, R. (2018). Suppression of EEG mu rhythm during action observation corresponds with subsequent changes in behavior. Brain research, 1691, 55-63.

Benjamini, Y., \& Hochberg, Y. (1995). Controlling the false discovery rate: A practical and powerful approach to multiple testing. Journal of the Royal Statistical Society. Series $B$ (Methodological), 57, 289-300.

Comalli, D. M., Keen, R., Abraham, E., Foo, V. J., Lee, M. H., \& Adolph, K. E. (2016). The development of tool use: Planning for end-state comfort. Developmental Psychology, 52, 1878-1892.

Daw, N. D., Niv, Y., \& Dayan, P. (2005). Uncertainty-based competition between prefrontal and dorsolateral striatal systems for behavioral control. Nature neuroscience, 8(12), 17041711.

Decker, J. H., Otto, A. R., Daw, N. D., \& Hartley, C. A. (2016). From creatures of habit to goaldirected learners: Tracking the developmental emergence of model-based reinforcement learning. Psychological Science, 27(6), 848-858.

Delorme, A., \& Makeig, S. (2004). EEGLAB: an open source toolbox for analysis of single-trial EEG dynamics including independent component analysis. Journal of neuroscience methods, 134(1), 9-21.

Franchak, J. M., Kretch, K. S., Soska, K. C., \& Adolph, K. E. (2011). Head-mounted eye tracking: A new method to describe infant looking. Child Development, 82, 1738-1750.

Fuelscher, I., Williams, J., Wilmut, K., Enticott, P. G., \& Hyde, C. (2016). Modeling the maturation of grip selection planning and action representation: Insights from typical and atypical motor development. Frontiers in Psychology, 7, 108.

Gibson, J. J. (1979). The ecological approach to visual perception. Boston, MA: Houghton Mifflin.

Keen, R. (2011). The development of problem solving in young children: A critical cognitive skill. Annual Review of Psychology, 62, 1-21.

Knudsen, B., Henning, A., Wunsch, K., Weigelt, M., \& Aschersleben, G. (2012). The end-state comfort effect in 3- to 8-year-old children in two object manipulation tasks. Frontiers in Psychology, 3, 1-10.

Krajenbrink, H., Lust, J., Wilson, P., \& Steenbergen, B. (2020). Development of motor planning in children: Disentangling elements of the planning process. Journal of Experimental Child Psychology, 199, 104945.

Kretch, K. S., \& Adolph, K. E. (2013a). Cliff or step? Posture-specific learning at the edge of a drop-off. Child Development, 84, 226-240.

Kretch, K. S., \& Adolph, K. E. (2013b). No bridge too high: Infants decide whether to cross based on the probability of falling not the severity of the potential fall. Developmental Science, 16, 336-351.

Land, W., Volchenkov, D., Bläsing, B. E., \& Schack, T. (2013). From action representation to action execution: exploring the links between cognitive and biomechanical levels of motor control. Frontiers in Computational Neuroscience, 7, 127.

Lee, M. H., Liu, Y. T., \& Newell, K. M. (2006). Longitudinal expressions of infant's prehension as a function of object properties. Infant Behavior and Development, 29, 481-493. 
Lockman, J. J., Fears, N. E., \& Jung, W. P. (2018). The development of object fitting: The dynamics of spatial coordination. In J. M. Plumert (Ed.), Advances in Child Development and Behavior (Vol. 55, pp. 31-72). Amsterdam: Elsevier.

Newell, A., \& Simon, H. A. (1972). Human problem solving. Englewood Cliffs, NJ: Prentice Hall.

Nussenbaum, K., \& Hartley, C. A. (2019). Reinforcement learning across development: What insights can we draw from a decade of research? Developmental Cognitive Neuroscience, 40, 100733.

Ossmy, O., Han, D., Cheng, M., Kaplan, B. E., \& Adolph, K. E. (2020). Look before you fit: The real-time planning cascade in children and adults. Journal of Experimental Child Psychology, 189, 104696.

Rosenbaum, D. A., Chapman, K. M., Weigelt, M., Weiss, D. J., \& van der Wel, R. (2012). Cognition, action, and object manipulation. Psychological Bulletin, 138, 924-946.

Rosenbaum, D. A., \& Feghhi, I. (2019). The time for action is at hand. Attention, Perception, \& Psychophysics.

Rosenbaum, D. A., Marchak, F., Barnes, H. J., Vaughan, J., Slotta, J., \& Jorgensen, M. (1990). Constraints for action selection: Overhand versus underhand grips. In M. Jeannerod (Ed.), Attention and Performance XIII: Motor representation and control (pp. 321-342). Hillsdale, NJ: Lawrence Erlbaum Associates.

Shutts, K., Ornkloo, H., von Hofsten, C., Keen, R., \& Spelke, E. S. (2009). Young children's representations of spatial and functional relations between objects. Child Development, 80, $1612-1627$.

Stockel, T., Hughes, C. M. L., \& Schack, T. (2012). Representation of grasp postures and anticipatory motor planning in children. Psychological Research, 76, 768-776.

Toussaint, L., Tahej, P.-K., Thibaut, J.-P., Possamai, C.-A., \& Badets, A. (2013). On the link between action planning and motor imagery: a developmental study. Experimental Brain Research, 231(3), 331-339.

van Hof, P., van der Kamp, J., \& Savelsbergh, G. J. P. (2008). The relation between infants' perception of catchableness and the control of catching. Developmental Psychology, 44, 182-194.

Willats, P. (1989). Development of problem-solving in infancy. In A. Slater \& G. Bremner (Eds.), Infant development. Hillsdale, NJ: Erlbaum.

Witherington, D. C. (2005). The development of prospective grasping control between 5 and 7 months: A longitudinal study. Infancy, 7, 143-161.

Wong, T. T. (2015). Performance evaluation of classification algorithms by k-fold and leave-oneout cross validation. Pattern Recognition, 48, 2839-2846.

Wunsch, K., Henning, A., Aschersleben, G., \& Weigelt, M. (2013). A systematic review of the end-state comfort effect in normally developing children and in children with developmental disorders. Journal of Motor Learning and Development, 1, 59-76.

Wunsch, K., Weiss, D. J., Schack, T., \& Weigelt, M. (2015). Second-order motor planning in children: Insights from a cup-manipulation-task. Psychological Research, 79, 669-677. 


\section{Supplemental Material}

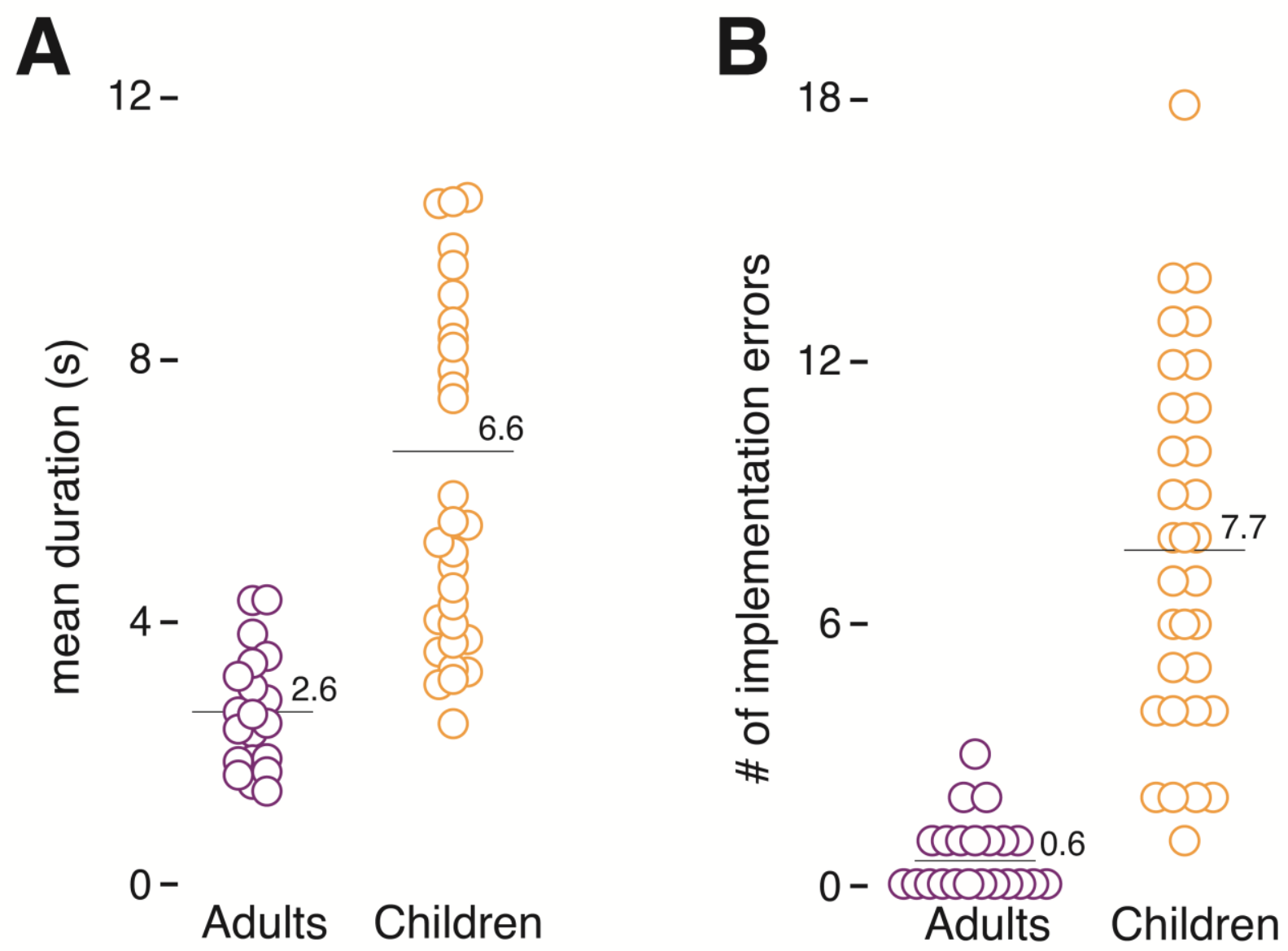

Figure S1. Measures of efficiency in adults and children. (A) Duration of non-dominant-hand trials (from screen opening to pounding the peg flat). Each symbol denotes mean trial time for each participant. Horizontal lines denote group means. (B) Number of implementation errors (failing to hit the peg) on nondominant-hand trials. Each symbol denotes total number of errors for each participant. Horizontal lines denote group means.

Video S1. Examples of implementation errors by one typical child and adult.

Video S2. Examples of last-moment grip decisions by one typical child and adult.

Video S3. Video demonstrating the probability of looking at various parts of the scene accumulated across each group (Left: adults; Right: adaptive child solvers; Left: nonadaptive child solvers). If the pixel is black at specific frame, then the group's probability of fixating that pixel is 0 (no participant looked at that area). As the probability increases, the pixel is clearer. When the probability is 1 , the pixel is shown in its entirety. Probability was calculated similarly to the heat map in Figure 5D. 